PROCEEDINGS OF THE

AMERICAN MATHEMATICAL SOCIETY

Volume 130, Number 9, Pages 2569-2577

S 0002-9939(02)06413-4

Article electronically published on February 12, 2002

\title{
LOCAL INDICABILITY IN ORDERED GROUPS: BRAIDS AND ELEMENTARY AMENABLE GROUPS
}

\author{
AKBAR RHEMTULLA AND DALE ROLFSEN
}

(Communicated by Stephen D. Smith)

\begin{abstract}
Groups which are locally indicable are also right-orderable, but not conversely. This paper considers a characterization of local indicability in right-ordered groups, the key concept being a property of right-ordered groups due to Conrad. Our methods answer a question regarding the Artin braid groups $B_{n}$ which are known to be right-orderable. The subgroups $P_{n}$ of pure braids enjoy an ordering which is invariant under multiplication on both sides, and it has been asked whether such an ordering of $P_{n}$ could extend to a rightinvariant ordering of $B_{n}$. We answer this in the negative. We also give another proof of a recent result of Linnell that for elementary amenable groups, the concepts of right-orderability and local indicability coincide.
\end{abstract}

\section{Definitions and Statement of Results}

A right-ordered group is a pair $(G,<)$, where $G$ is a group, $<$ is a strict total ordering of the elements of $G$, and right-invariance holds:

$$
g<h \Rightarrow g k<h k, \forall g, h, k \in G .
$$

If the ordering is also left-invariant,

$$
g<h \Rightarrow k g<k h, \forall g, h, k \in G,
$$

then we call $(G,<)$ a bi-ordered group (also known in the literature as "totally ordered" or, simply, "ordered").

A group $G$ is said to be locally indicable if for every nontrivial finitely-generated subgroup $H$ of $G$ there is a nontrivial homomorphism $H \rightarrow \mathbf{Z}$ onto the additive group of integers.

Proposition 1.1. Locally indicable groups are right-orderable.

Proof. Burns and Hale in [4] show that a group $G$ is right-orderable if and only if every finitely generated nontrivial subgroup of $G$ has a nontrivial quotient that is also right-orderable. See [16], Theorem 7.3.1, or [13], Theorem 3.2.1. Since $\mathbf{Z}$ is right-orderable, the proposition follows.

The converse is not true. Bergman [2] gave an example of a finitely-generated right-orderable group which is perfect - its abelianization is trivial. Any homomorphism of such a group to $\mathbf{Z}$ must be trivial, so it is not locally indicable.

Received by the editors February 16, 2001 and, in revised form, April 26, 2001.

2000 Mathematics Subject Classification. Primary 20F36; Secondary 20F60, 06F15.

The authors thank NSERC for partial financial support. 
The class of elementary amenable groups is the smallest class of groups which contains all finite groups, all abelian groups and is closed under taking subgroups, extensions, factor groups and unions of directed systems of such groups. This class is strictly larger than the class of solvable groups, but properly contained in the class of all amenable groups. We shall show how the following result, first proved by Linnell [14 in 1999, follows from previously-known results.

Theorem 1.2. If $G$ is an elementary amenable group, then $G$ is locally indicable if and only if $G$ is right-orderable.

This will be shown in Section 5. A different criterion will be proven first, in Section 2. It has applications to the theory of Artin's braid groups. In fact, this application was the original motivation for the present work.

Theorem 1.3. Suppose $(G,<)$ is a right-ordered group and there is a finite-index subgroup $H$ of $G$ such that $(H,<)$ is a bi-ordered group. Then $G$ is locally indicable.

Corollary 1.4. If $(G,<)$ is a bi-ordered group, then $G$ is locally indicable.

The braid groups $B_{n}$ and their finite-index subgroups $P_{n}$ of pure braids will be discussed in Section 3, where we will prove the following consequence of Theorem 1.3. It should be noted that $P_{n}$ is bi-orderable, whereas $B_{n}$ is only right-orderable (for $n \geq 3$ ).

Corollary 1.5. If $n \geq 5$ and $<$ is a right-invariant ordering on $B_{n}$, then $\left(P_{n},<\right)$ cannot be a bi-ordered group. The same is true for any finite-index subgroup in place of $P_{n}$.

\section{ConRAD-TYPE ORDERINGS}

Right-orderable groups are also left-orderable, but by a different ordering (compare inverses). There are many groups which are right-orderable, but not biorderable, a simple example being the Klein bottle group with two generators $x$ and $y$ and the relation $y x y^{-1}=x^{-1}$. The reader is referred to [16] or [13] for further information on ordered groups.

The theory of ordered groups is well over a century old. Indeed, we will make use of a 1902 result of Hölder, as generalized by Conrad [7], regarding the structure of ordered groups. An ordering $<$ of a group $G$ is said to be Archimedian if for every $x$ and $y$ in $G$ with $e<x<y$, there exists a positive integer $N$ such that $y<x^{N}$.

Theorem 2.1 (Hölder). If $(G,<)$ is an Archimedian ordered group, then there is an order-preserving algebraic isomorphism of $G$ with a subgroup of the additive real numbers. In particular, $G$ is abelian.

We remark that Hölder's theorem also holds for right-orderable groups, as shown by Conrad. More generally, Conrad investigated the structure of arbitrary rightordered groups, and defined a useful concept which lies between right-invariance and bi-invariance.

A right-ordered group $(G,<)$ is said to be of Conrad type if for all $a, b \in G$, with $e<a, e<b$ there exists a positive integer $N$ such that $a<a^{N} b$. The following criterion is perhaps more natural. We use the notation $x<<y$ to mean $x^{N}<y$ for all integers $N$. 
Lemma 2.2. In a right-ordered group $(G,<)$ the ordering is of Conrad type if and only if for all $x, y \in G$,

$$
e<x<<y \quad \Rightarrow \quad y^{-1}<x^{-1} .
$$

Proof. The proof is completely routine, using the substitutions $a=x$ and $b=y^{-1} x$, and left to the reader.

Lemma 2.3. A bi-ordered group is of Conrad type.

Proof. In a bi-ordered group we use left- and then right-invariance to see

$$
g<h \Leftrightarrow h^{-1}<g^{-1} .
$$

In fact, this is a necessary and sufficient condition for a right-ordered group to be bi-ordered.

Theorem 2.4. Let $(G,<)$ be a right-ordered group and suppose $H$ is a subgroup of $G$ of finite index. If $(H,<)$ is of Conrad type, then so is $(G,<)$.

Proof. Assume the hypothesis, but that $(G,<)$ is not of Conrad type. Then there exist $a, b \in G$ with $e<a$ and $e<b$ and $a^{N} b<a$ for all positive $N$. First note that $a>b$, for $a>e$ implies $a b>b$ and therefore $a>a b>b$. Next note that $a>a^{N} b$ implies $a>a b>a^{N} b^{2}$, and a simple induction shows $a>a^{N} b^{M}$ for all integers $N \geq 0, M \geq 1$. Since $H$ is of finite index, there is a positive integer $K$ such that $a^{K}$ and $b^{K}$ belong to $H$. But then we have $\left(a^{K}\right)^{N} b^{K}<a<a^{K}$, which contradicts the assumption that the ordering has Conrad type on $H$.

In Section 6 we will see that the hypothesis of finite index can be weakened considerably in the theorem above.

Proposition 2.5. Assume $(G,<)$ is a right-ordered group which is NOT of Conrad type and that $a, b \in G$ violate the Conrad condition, i.e. they satisfy $e<a, e<b$ and $a>a^{N} b$ for all positive integers $N$. Then we have $v a>w b$ for any words $v, w$ in the semigroup $S$ generated by $a$ and $b$.

Proof. Since $v>e$ implies $v a>a$, it suffices to show that $a>w b$. If $w=$ $a^{N} b^{M}, N, M \geq 0$, this follows as in the proof of the previous theorem. Otherwise, write $w=a^{N} b^{M} w^{\prime}$ where $w^{\prime} \in S, N \geq 0, M \geq 1$. Then $a>a^{N} b^{M}$ implies $a w^{\prime} b>$ $w b$ and we may assume inductively (on the total exponent of $b$ ) that $a>a w^{\prime} b$. Thus $a>w b$.

Corollary 2.6. A right-orderable group which is not of Conrad type must contain a free sub-semigroup with two generators.

Proof. Consider the semigroup $S$ of the proposition, generated by $a$ and $b$. It must be free: consider two nonempty words in $a, b$ with only positive exponents. Since we are in a group, we may assume one word ends in $a$ while the other ends in $b$. The proposition shows they cannot be equal.

Suppose $(G,<)$ is a right-ordered group. A subgroup $H$ of $G$ is said to be convex if $h<g<h^{\prime}, h, h^{\prime} \in H$ implies $g \in H$. The set of all convex subgroups of $G$ is ordered by inclusion, and closed under arbitrary unions and intersections. Suppose $C^{\prime}$ and $C$ are distinct convex subgroups of $G$ such that $C^{\prime} \subset C$, and the only convex subgroups $H$ of $G$ satisfying $C^{\prime} \subset H \subset C$ are $H=C$ and $H=C^{\prime}$. Then the pair $\left(C^{\prime}, C\right)$ is called a convex jump. 
Theorem 2.7 (Conrad). Suppose $(G,<)$ is a right-ordered group of Conrad type, and that $\left(C^{\prime}, C\right)$ is a convex jump in $G$. Then $C^{\prime}$ is normal in $C$ and $C / C^{\prime}$ is isomorphic (in both the algebraic and order sense) with a subgroup of the additive real numbers.

Corollary 2.8. If $G$ is finitely generated and Conrad right-ordered, then there is a homomorphism of $G$ onto a nontrivial direct product of infinite cyclic groups.

Proof. Let $\Gamma$ be a generating set for $\mathrm{G}$, which we may assume to be minimal (i.e. not redundant) and to consist of positive elements. Let $g \in \Gamma$ be the greatest element, relative to the given ordering of $G$. Let $C^{\prime}$ be the union of all the convex subgroups of $G$ which do not contain $g$. It is easily verified that $\left(C^{\prime}, G\right)$ is a convex jump in $G$. By Theorem 2.7, $G / C^{\prime}$ is isomorphic with a subgroup of the real numbers, so in particular it is a nontrivial finitely-generated torsion-free abelian group. By the fundamental theorem of abelian groups, the projection $G \rightarrow G / C^{\prime}$ satisfies the conclusion.

Theorem 2.9. If $(G,<)$ is a right-ordered group of Conrad type, then $G$ is locally indicable.

Proof. Let $H$ be a finitely generated subgroup of $G$. Considering $H$ itself as a Conrad right-ordered group, there is, by the above corollary, a nontrivial homomorphism $H \rightarrow Z^{n}$ and therefore, after an appropriate composition, a surjection $H \rightarrow Z$.

The converse of this result will be discussed in Section 4 .

\section{Application to Artin's braid Groups}

We recall that the full Artin braid group $B_{\infty}$ has generators $\sigma_{1}, \sigma_{2}, \ldots$ and relations

$$
\sigma_{i} \sigma_{j}=\sigma_{j} \sigma_{i},|i-j|>1, \quad \sigma_{i} \sigma_{i+1} \sigma_{i}=\sigma_{i+1} \sigma_{i} \sigma_{i+1} .
$$

The $n$-string braid group $B_{n}, n>1$, can be considered the subgroup generated by $\sigma_{1}, \ldots, \sigma_{n-1}$. If one adjoins the relations $\sigma_{i}^{2}=1$, this defines a mapping of $B_{n}$ onto the symmetric group $\Sigma_{n}$. Its kernel is the pure braid group $P_{n}$. Clearly $P_{n}$ is a normal subgroup of $B_{n}$ of index $n$ ! The following is well-known.

Theorem 3.1. For all $n \geq 5$, the braid groups $B_{n}$ are not locally indicable.

Proof. According to Gorin and Lin [11], for $n \geq 5$, the commutator subgroup $\left[B_{n}, B_{n}\right]$ is finitely-generated and perfect. Any homomorphism of $\left[B_{n}, B_{n}\right]$ to an abelian group must be trivial, so $B_{n}$ is not locally indicable.

We now turn to the proof of Corollary 1.5, It will follow immediately, noting Lemma 2.3. from the following stronger result.

Theorem 3.2. Let $n \geq 5$, and suppose $<$ is an ordering such that $\left(B_{n},<\right)$ is a right-ordered group. Then the ordering restricted to any finite index subgroup of $B_{n}$ is not of Conrad type.

Proof. According to Theorems 3.1 and 2.9, the ordering of $B_{n}$ cannot be of Conrad type. Then apply Theorem 2.4. 
We close this section with a discussion of which braid groups can have a Conrad type right-ordering. If $n \geq 5$, we have seen already that there is no possibility of givinging $B_{n}$ a Conrad ordering.

Proposition 3.3. The Dehornoy ordering on $B_{n}, n \geq 3$, is not of Conrad type.

Proof. First, we must describe Dehornoy's ordering: it suffices to declare when a braid $\beta$ is positive, i.e. $e<\beta$. The criterion is that $\beta$ is positive iff there is an expression in the generators $\sigma_{i}$ such that the generator with lowest subscript appears with only positive exponent. Now we let $\alpha=\sigma_{1}$ and $\beta=\sigma_{1} \sigma_{2}^{-1}$, clearly both Dehornoy-positive. Then repeated use of the identity $\sigma_{1} \sigma_{2}^{-1} \sigma_{1}^{-1}=\sigma_{2}^{-1} \sigma_{1}^{-1} \sigma_{2}$ shows that

$$
\alpha^{N} \beta \alpha^{-1}=\sigma_{1}^{N+1} \sigma_{2}^{-1} \sigma_{1}^{-1}=\sigma_{2}^{-1} \sigma_{1}^{-1} \sigma_{2}^{N+1} .
$$

Since its inverse is positive, we have $\alpha^{N} \beta \alpha^{-1}<e$ and therefore $\alpha^{N} \beta<\alpha$ for all $N$, in Dehornoy's ordering, showing that it is not of Conrad type.

Theorem 3.4. $B_{3}$ and $B_{4}$ can be given right-orderings of Conrad type, and are therefore locally indicable.

The following simple lemma says that the category of Conrad-right-orderable groups is closed under extensions. The Conrad property is clearly also inherited by subgroups, and preserved under directed unions.

Lemma 3.5. Suppose $1 \rightarrow A \rightarrow B \rightarrow C \rightarrow 1$ is an exact sequence of groups, with $A$ and $C$ having right-orderings of Conrad type. Define an ordering on $B$, by $b_{1}<b_{2}$ if their respective images $c_{1}, c_{2}$ satisfy $c_{1}<c_{2}$ in $C$, or else $c=c^{\prime}$ and $e<b_{2} b_{1}^{-1} \in A$. Then this is a right-ordering of $B$ of Conrad type.

The proof is routine, and left to the reader.

Proof of Theorem 3.4 The map $B_{3} \rightarrow \mathbf{Z}$ which takes a braid to its exponent sum when expressed as a word in the $\sigma_{i}$, can be identified with the abelianization map. The commutator subgroup is a free group $F_{2}$ of rank two. One way to see this is to recall that $B_{3}$ is isomorphic with the fundamental group of the complement of the trefoil knot, which is known to fibre over a circle, with fibre a punctured torus; see for example [18, p. 327. It follows that we have an exact sequence

$$
1 \rightarrow F_{2} \rightarrow B_{3} \rightarrow Z \rightarrow 1
$$

Since $F_{2}$ and $Z$ are bi-orderable, they certainly have Conrad right-orderings, which by the lemma induces one on $B_{3}$.

Now to consider $B_{4}$ we employ a bootstrap trick. There is a homomorphism $B_{4} \rightarrow B_{3}$ defined by

$$
\sigma_{1} \rightarrow \sigma_{1}, \quad \sigma_{2} \rightarrow \sigma_{2}, \quad \sigma_{3} \rightarrow \sigma_{1}
$$

Its kernel $K$, the normal closure of $\sigma_{1} \sigma_{3}^{-1}$, is contained in the commutator subgroup of $B_{4}$. According to Gorin and Lin, this commutator subgroup is a semidirect product of two copies of $F_{2}$. By Lemma 3.5, this is Conrad right-orderable, and therefore so is $K$. Note the following exact sequence:

$$
1 \rightarrow K \rightarrow B_{4} \rightarrow B_{3} \rightarrow 1 \text {. }
$$

Another application of the lemma shows that $B_{4}$ is Conrad right-orderable, completing the proof. 


\section{CharaCterizing LOCAL INDICABILITY}

We have already seen that if a group has a right-ordering of Conrad type, then it is locally indicable. The converse is also true. A different argument appears in [10]. We offer a version which follows as a corollary of Theorem 3 of Brodskii in [3].

Theorem 4.1. A group is locally indicable if and only if it admits a right-ordering of Conrad type.

Proof. By Theorem 3 of [3], for any quasivariety $\mathcal{V}$, the class of locally $\mathcal{V}$ indicable groups coincides with the class of groups having a normal system with factors in the class $\mathcal{V}$. Now, taking $\mathcal{V}$ to be the quasivariety of all torsion-free Abelian groups, we see that the class of locally indicable groups coincides with the class of groups having a normal system with torsion-free Abelian factors.

We recall the definition of a normal system. A chain of subgroups $\Sigma$ of a group $G$ is a normal system with factors in $\mathcal{V}$ if $(i)\{e\} \in \Sigma,(i i) G \in \Sigma$, (iii) $\Sigma$ contains all unions and intersections of its members ( $\Sigma$ is complete) and (iv) if $H \in \Sigma$ has an immediate successor $K \in \Sigma$ in the natural ordering of $\Sigma$, then $H \triangleleft K$ and $K / H \in \mathcal{V}$.

For any element $x \neq 1$ in $G$, let $\vee_{x}$ be the union of all members of $\Sigma$ not containing $x$ and let $\wedge_{x}$ be the intersection of all members of $\Sigma$ containing $x$. Then $\vee_{x}, \wedge_{x}$ are members of $\Sigma$ by $(i i i), \vee_{x} \triangleleft \wedge_{x} \in \Sigma$ and $\wedge_{x} / \vee_{x} \in \mathcal{V}$ by $(i v)$. The pair $\left(\vee_{x}, \wedge_{x}\right)$ is called a jump in $\Sigma$.

A group $G$ having a normal system $\Sigma$ with torsion-free Abelian factors may be given a right-ordering of Conrad type as follows. For each jump $(\vee, \wedge)$ in $\Sigma$ totally order the torsion free abelian group $\wedge / \vee$. For an element $1 \neq x \in G$, put $x$ in the positive cone $P$ of $G$ if $x \vee_{x}$ is positive in $\wedge_{x} / \vee_{x}$. The set $P$ has the properties: $P \cap P^{-1}=\Phi, P P \subseteq P$, and $P \cup P^{-1}=G \backslash\{e\}$. That $P$ is the positive cone of a Conrad order follows from the fact every two sided order is a Conrad right-order. Observe that under this order on $G$ every member of $\Sigma$ is a convex subgroup, but $\Sigma$ need not coincide with the set of all convex subgroups.

\section{Elementary amenable Groups}

In this section we prove Theorem 1.2, that any elementary amenable rightorderable group is locally indicable. We note that Linnell [14] has proved this result by different means. Let $N F S$ denote the class of groups $G$ that do NOT contain a free sub-semigroup with two generators. According to Corollary [2.6, every right-ordering of a group in NFS must be of Conrad type. The following result of Longobardi, Maj and Rhemtulla [15] is the key to our argument.

Theorem 5.1. Suppose the right-ordered group $G$ is the union of an ascending well-ordered family of subgroups $\left\{H_{\alpha}\right\}$ with the property that for each $\alpha$, the quotient $H_{\alpha+1} / H_{\alpha}$ belongs to NFS. Then $G$ is locally indicable.

Recall that $\Sigma=\left\{H_{\alpha} ; \alpha \in \mathcal{A}\right\}$ is an ascending series of $G$ with factors in $\mathcal{V}$ if $\{e\}, G \in \Sigma, \mathcal{A}$ is a well ordered set, for all $\alpha, \beta \in \mathcal{A}, \alpha<\beta \Rightarrow H_{\alpha}<H_{\beta}, H_{\alpha} \triangleleft H_{\alpha+1}$, and $H_{\alpha+1} / H_{\alpha} \in \mathcal{V}$.

Lemma 5.2. A group $G$ is elementary amenable if and only if $G$ is the union of an ascending well-ordered family of subgroups $\left\{H_{\alpha}\right\}$ with the property that for each $\alpha$, the quotient $H_{\alpha+1} / H_{\alpha}$ is abelian-by-finite. 
Proof. Recall that the class elementary amenable groups is the smallest class of groups which contains all abelian-by-finite groups, is closed under group extensions and is closed under directed unions. Proof of this lemma is due to Chou (Proposition 2.2 , [6]), and it is also given in [20].

Lemma 5.3. Every group which is abelian-by-finite belongs to NFS.

Proof. Let $H$ be a group with abelian normal subgroup $N$, and $H / N$ finite. If $g, h \in$ $H$, then for some positive integer $k$ we have $g^{k}, h^{k} \in N$, and hence $g^{k} h^{k}=h^{k} g^{k}$, so that the subsemigroup generated by $g$ and $h$ is not free. Therefore $H \in N F S$.

Corollary 5.4. For elementary amenable groups, right-orderability is equivalent to local indicability.

\section{Subgroups}

We finish with a generalization of Theorem 2.4.

Let $X$ be a class of groups that is subgroup and quotient closed and with the property that if $G \in$ Abelian-by- $X$ and $G$ is right-orderable, then $G$ is locally indicable.

Examples of such classes are $X=$ the class of all finite groups and $X=$ the class of solvable groups [5]. Another example is the class $X$ of groups $G$ which have an ascending series of normal subgroups $\left\{G_{\alpha}\right\}$ such that $G_{\alpha+1} / G_{\alpha} \in N F S$ (see [15]).

Theorem 6.1. Suppose $G$ is right-orderable, $N$ is a normal subgroup of $G$ and $G / N \in X$. Then either $G$ is locally indicable, or for every right-order $<$ on $G$, the restriction of $<$ to $N$ is NOT of Conrad type.

Proof. Suppose that $G$ is not locally indicable and $\leq$ is a right-order on $G$ such that its restriction $(N, \leq)$ to the subgroup $N$ is of Conrad type. Let $G_{1}$ be a finitely generated subgroup of $G$ such that $G_{1} / G_{1}{ }^{\prime}$ is finite. Such a subgroup $G_{1}$ exists since $G$ is not locally indicable.

Now $G_{1} /\left(N \cap G_{1}\right) \cong N G_{1} / N$ is a subgroup of $G / N$ so it is an $X$ group. Moreover the order $\leq$ on $G_{1}$ is not of Conrad type since $G_{1} / G_{1}{ }^{\prime}$ is finite, but its restriction to $N \cap G_{1}$ is a Conrad right-order. Thus pass to $G_{1}$ if necessary and assume that $G$ is finitely generated and $G / G^{\prime}$ is finite.

If $N$ is bounded above or below under $\leq$ (we say $N$ is bounded above if there exists some element $x$ in $G$ such that $n \leq x, \forall n \in N)$, then by Lemma 4 of [15], there is a proper normal relatively convex subgroup $C$ of $G$ where $C \geq N$. Then $G / C$ is a right-orderable $X$ group and hence locally indicable by hypothesis. Thus $G / C$ has a torsion-free Abelian quotient and $G / G^{\prime} C$ is not finite, a contradiction.

Thus assume $N$ is not bounded. By this we mean that for any elements $x \leq y$ in $G$, there exist $n, m \in N$ such that $n \leq x \leq y \leq m$.

Now let $\left\{C_{\lambda}, \lambda \in \Lambda\right\}$ be the set of all proper convex subgroups of $N$ under $\leq$. For each $C_{\lambda}$, let $H_{\lambda}$ be the intersection of all convex subgroups of $(G, \leq)$ that contain $C_{\lambda}$. Note that $H_{\lambda}$ is convex since intersection of an arbitrary set of convex subgroups is convex. Indeed $H_{\lambda}$ is the smallest convex subgroup of $(G, \leq)$ such that $H_{\lambda} \cap N=C_{\lambda}$.

Next consider the set $\left\{H_{\lambda}, \lambda \in \Lambda\right\}$. Let $H=\bigcup_{\lambda \in \Lambda} H_{\lambda}$. Then $H \neq G$ since $G$ is finitely generated, each $H_{\lambda}$ is a proper subgroup of $G$ and the set $\left\{H_{\lambda}, \lambda \in \Lambda\right\}$ is linearly ordered. 
Let $C=H \cap N$. Then $C$ is a proper convex subgroup of $N, C \geq C_{\lambda}, \lambda \in \Lambda$. So $(C, N)$ is a jump.

We now show that $C$ is normal in $G$. Since $(N, \leq)$ is of Conrad type by assumption, it follows from Theorem [2.7 that $C \triangleleft N$ and $N / C$ is torsion-free Abelian. Thus for every $a \in N \backslash C, e \leq a$, the set $\left\{a^{n} ; n \in \mathbf{Z}\right\}$ is unbounded in $N$ and we have shown that $N$ is unbounded in $G$. Hence $g^{-1} a g>e$ for every $g \in G$ (15, Lemma $3)$. If $g^{-1} a g \in C$, then $\left(g^{-1} a g\right)^{n} \leq a \quad \forall n \geq 1$. Hence $b_{n}=a g^{-1} a^{-n} g \geq e$ and $b_{n} \in N \backslash C$ and so the subgroup generated by $b_{n}$ is unbounded. Hence $g b_{n} g^{-1} \geq e$.

From $e \leq g b_{n} g^{-1}=g\left(a g^{-1} a^{-n} g\right) g^{-1}=g a g^{-1} a^{-n}$ we get $a^{n} \leq g a g^{-1}, \forall n \geq 1$. But this contradicts the statement that the set $\left\{a^{n} ; n \in \mathbf{Z}\right\}$ is unbounded. Thus the set $N \backslash C$ is normal in $G$ and hence $C \triangleleft G$.

$C$ is bounded above in $G$ since it is bounded above in $N$. So, by ([15], Lemma 4), there is a proper normal relatively convex subgroup $K$ of $G$ containing $C$. So $G / K$ is a right-orderable group and it is an Abelian-by- $X$ group so that, by hypothesis, it is locally indicable. Since it is finitely generated, it has a torsion-free Abelian quotient.

For an application of Theorem 6.1 we return to braid groups $B_{n}, n \geq 5$. The subgroups $P_{n}$ of pure braids are known to be orderable. Even more, they are known to be residually torsion-free nilpotent [19]; and as such, their descending central series $\Gamma_{i}\left(P_{n}\right), i=1,2, \ldots$, intersect in the unit group $\{1\}$. The subgroups $\Gamma_{i}\left(P_{n}\right)$ are defined inductively by $\Gamma_{1}\left(P_{n}\right)=P_{n}$ and $\Gamma_{i+1}\left(P_{n}\right)=\left[\Gamma_{i}\left(P_{n}\right), P_{n}\right]$. As a consequence of the above theorem, we can say that for any right-order on $B_{n}$, its restriction to $\Gamma_{i}\left(P_{n}\right)$ is not of Conrad type for all values of $i$. A similar statement holds with the terms of the descending central series replaced by the terms of the derived series of $P_{n}$.

\section{REFERENCES}

1. Emil Artin, Theorie der Zöpfe, Abh. Math. Sem. Univ. Hamburg 4 (1925), 47-72.

2. George Bergman, Right-orderable groups that are not locally indicable, Pacific J. Math. 147 (1991), 243-248. MR 92e:20030

3. S. D. Brodskii, Equations over groups, and groups with one defining relation, Sibirski Matematicheskii Zhurnal 25(1984), 84-103. MR 86e:20026

4. R. G. Burns and V. W. Hale, A note on group rings of certain torsion-free groups, Canad. Math. Bull. 15 (1972), 441-445. MR 46:9149

5. I. M. Chiswell and P. H. Kropholler, Soluble right-orderable groups are locally indicable, Canad. Math. Bull. 36 (1993), 22-29. MR 93j:20088

6. Ching Chou, Elementary amenable groups, Illinois J. Math. 24 (1980), 396-407. MR 81h:43004

7. P. F. Conrad, Right-Ordered Groups, Michigan Math. J. 6 (1959), 267-275. MR 21:5684

8. Patrick Dehornoy, From large cardinals to braids via distributive algebra, J. Knot Theory Ramifications 4 (1995), no. 1, 33-79. MR 96g:20056

9. Roger Fenn, Michael Greene, Dale Rolfsen, Colin Rourke, and Bert Wiest, Ordering the braid groups, Pacific J. Math. 191 (1999), 49-74. MR 2000j:20064

10. A. M. W. Glass, Partially Ordered groups, Series in Algebra, vol. 7, World Scientific, London, 1999. MR 2001g:06002

11. E. A. Gorin and V. Ya. Lin, Algebraic equations with continuous coefficients and some problems of the algebraic theory of braids, Math. USSR Sbornik 7 (1969), 569-596. MR 40:4939

12. Djun M. Kim and Dale Rolfsen, Ordering groups of pure braids and hyperplane arrangements, preprint.

13. Valeriŭ M. Kopitov and Nikolaŭ Ya. Medvedev, Right-Ordered Groups, Plenum Publishing Corporation, New York, 1996. MR 97h:06024a 
14. Peter A. Linnell, Left ordered amenable and locally indicable groups, J. London Math. Soc. 60 (1999), 133-142. MR 2001c:20092

15. Patrizia Longobardi, Mercede Maj and Akbar Rhemtulla, When is a right-orderable group locally indicable, Proc. Amer. Math. Soc. 128 (2000), 637-641. MR 2000j:20072

16. Roberta Mura and Akbar Rhemtulla, Orderable groups, Lecture Notes in Pure and Applied Mathematics, vol. 27, Marcel Dekker, New York, 1977. MR 58:10652

17. L. P. Neuwirth, The status of some problems related to knot groups, Topology Conference (Virginia Polytech. Inst. and State Univ., Blacksburg, Va., 1973), Springer, Berlin, 1974, pp. 209-230. Lecture Notes in Math., Vol. 375. MR 55:4129]

18. Dale Rolfsen, Knots and Links, Publish or Perish, Inc., Houston, 1990. MR 95c:57018

19. Dale Rolfsen and Jun Zhu, Braids, orderings and zero divisors, J. Knot Theory and Ramifications 7 (1998), 837-841. MR 99g:20072

20. S. Wagon, The Banach-Tarski paradox, Cambridge University Press, 1993. MR 94g:04005

Department of Mathematical Sciences, University of Alberta, Edmonton, Alberta, CANADA T6G 2G1

E-mail address: ar@ualberta.ca

Department of Mathematics, University of British Columbia, Vancouver, British Columbia, Canada V6T $1 \mathrm{Z} 2$

E-mail address: rolfsen@math.ubc.ca 